Received Date: 13-Jul-2016

Revised Date: 22-Sep-2016

Accepted Date: 24-Oct-2016

Article Type: Research Report

\title{
Effect of interstimulus interval on cortical proprioceptive responses to passive finger movements
}

\author{
Eero Smeds $^{\mathrm{a}, \mathrm{b}}$, Harri Piitulainen ${ }^{\mathrm{a}, \mathrm{b}}$, Mathieu Bourguignon ${ }^{\mathrm{a}, \mathrm{b}, \mathrm{c}}$, Veikko Jousmäki ${ }^{\mathrm{a}, \mathrm{b}}$, \\ and Riitta Hari ${ }^{\mathrm{a}, \mathrm{b}, \mathrm{d}}$
}

${ }^{a}$ Department of Neuroscience and Biomedical Engineering, Aalto University, PO Box 12200, 00076 AALTO, Espoo, Finland

${ }^{b}$ Aalto Neurolmaging, Aalto University, 00076 AALTO, Espoo, Finland

${ }^{c} B C B L$, Basque Center on Cognition, Brain and Language, 20009 San Sebastian, Spain

${ }^{d}$ Department of Art, Aalto University, PO Box 31000, 00076 AALTO, Helsinki, Finland

\section{Corresponding author:}

Eero Smeds, MD

Department of Neuroscience and Biomedical Engineering, Aalto University

PO BOX 12200, 00076 AALTO, Espoo, Finland

Tel. +358 503556 189, Fax.+358 9470 23182, E-mail: eero.smeds@helsinki.fi

Proposed journal section: Cognitive Neuroscience

This article has been accepted for publication and undergone full peer review but has not been through the copyediting, typesetting, pagination and proofreading process, which may lead to differences between this version and the Version of Record. Please cite this article as doi: 10.1111/ejn.13447

This article is protected by copyright. All rights reserved. 
Running title: Effect of ISI on cortical proprioceptive responses

Key words: humans; magnetoencephalography; muscle stretch receptors; proprioception; primary somatosensory cortex

\begin{abstract}
Shortening of the interstimulus interval (ISI) generally leads to attenuation of cortical sensory responses. For proprioception, however, this ISI effect is still poorly known. Our aim was to characterize the ISI dependence of movement-evoked proprioceptive cortical responses and to find the optimum ISI for proprioceptive stimulation. We measured, from 15 healthy adults, magnetoencephalographic responses to passive flexion and extension movements of the right index finger. The movements were generated by a movement actuator at fixed ISIs of $0.5,1,2,4,8$, and $16 \mathrm{~s}$, in separate blocks. The responses peaked at $\sim 70 \mathrm{~ms}$ (extension) and $\sim 90 \mathrm{~ms}$ (flexion) in the contralateral primary somatosensory cortex. The strength of the cortical source increased with the ISI, plateauing at the 8-s ISI. Modeling the ISI dependence with an exponential saturation function revealed response lifetimes of $1.3 \mathrm{~s}$ (extension) and $2.2 \mathrm{~s}$ (flexion), implying that the maximum signal-to-noise ratio (SNR) in a given measurement time is achieved with ISIs of $1.7 \mathrm{~s}$ and $2.8 \mathrm{~s}$, respectively. We conclude that ISIs of 1.5-3 s should be used to maximize SNR in recordings of proprioceptive cortical responses to passive finger movements. Our findings can benefit the assessment of proprioceptive afference in both clinical and research settings.
\end{abstract}

\title{
Introduction
}

Proprioceptors in muscles, tendons, and joints sense positions, movements, and forces of body parts (for a review, see Proske \& Gandevia, 2012), thus being crucial for proper motor control (Scott, 2012). Previous studies have assessed proprioceptive afference by recording cortical responses to passive movements with electroencephalography (EEG; Rodin et al., 1969; Papakostopoulos et al., 1974; Shibasaki et al., 1980) and magnetoencephalography (MEG; Xiang et al., 1997; Lange et al., 2001; Alary et al., 2002; Druschky et al., 2003; Piitulainen et al., 2013, 2015; Bourguignon et al., 2015). These responses indeed seem to represent proprioceptive rather than tactile afference, as they can be measured even during cutaneous anesthesia (Starr et al., 1981; Abbruzzese et al., 1985; Mima et al., 1996) and regardless of the level of tactile component in the stimulation (Piitulainen et al., 2013; Bourguignon et al., 2015).

Transient passive movements elicit prominent responses peaking at $~ 70-80 \mathrm{~ms}$. According to MEG studies, these responses originate in the primary sensorimotor cortex (Xiang et al., 1997; Piitulainen et al., 2013), with some results highlighting the primary motor (MI) cortex (Lange et al., 2001) and others the primary somatosensory (SI) cortex (Alary et al., 2002).

This article is protected by copyright. All rights reserved. 
In previous studies on proprioceptive afference, the dependence of response amplitude on interstimulus interval (ISI) has received little attention. Yet, this information would be highly relevant for understanding the temporal operating scales of cortical proprioceptive processing. Furthermore, it would help develop reliable neurophysiological tests to quantify proprioceptive afference. Such robust tests would benefit both basic research and clinical evaluation of proprioceptive processing; the current clinical tests are quite poorly controlled as the examiner typically manually moves the patient's toes or fingers and asks the patient to report, eyes closed, the perceived changes in position.

As a general feature of sensory processing, shortening the ISI leads to attenuation of cortical responses. Maximum responses may be elicited only at ISIs of several seconds or even tens of seconds, depending on the response latency, cortical area, and sensory modality. Thus, previous stimuli can leave to the cortex long-lasting traces, the durations of which can be characterized with the "lifetime" of the response in question. The lifetime is the exponential decay constant of the exponential saturation function fitted to the peak response amplitudes as a function of ISI (Lu et al., 1992a). Lifetimes are typically shorter for short- than long-latency responses and shorter at lower than higher stages of the cortical processing stream.

The ISI effects have been scrutinized by means of MEG, e.g., in the auditory, visual, and somatosensory modalities. The supratemporal 100-ms auditory evoked field is attenuated at ISIs below 8-16 s and has a lifetime of 1.5 s (Hari et al., 1982; Lu et al., 1992a; Mäkelä et al., 1993). Occipital visual evoked fields peaking at $90-180 \mathrm{~ms}$ have lifetimes from $0.2 \mathrm{~s}$ to $0.6 \mathrm{~s}$ whereas the lifetimes for later extra-occipital responses at 180-440 ms range from 7 to $30 \mathrm{~s}$ (Uusitalo et al., 1996). In the SI cortex, shortening the ISI of median nerve stimulation from 0.5 to $0.2 \mathrm{~s}$ suppresses the 27-ms deflection P27m of the somatosensory evoked field (SEF) much more than the 20-ms deflection N20m (Tiihonen et al., 1989). Furthermore, the 35- and 60-ms SEFs attenuate monotonously when the ISI is reduced from 5 to $0.3 \mathrm{~s}$ (Wikström et al., 1996). In the secondary somatosensory (SII) cortex, the ISI dependence of the 100 -ms response resembles that of the auditory 100-ms response (Hari et al., 1993). These observations support the existence of multiple hierarchically organized timescales both within brain areas and within cortical processing streams (for a review, see Hari et al., 2010).

In previous studies on proprioceptive input, the 80-ms EEG responses to wrist extension decreased by $\sim 50 \%$ when the ISI was reduced from 6.7 to $1 \mathrm{~s}$ (Abbruzzese et al., 1985), and the 90ms EEG response to ankle flexion decreased by more than $50 \%$ when the ISI was reduced from 5 to $0.5 \mathrm{~s}$ (Starr et al., 1981). Also in a recent MEG study (Piitulainen et al., 2015), the 90-ms response to finger flexion-extension movements decreased by $80 \%$ when intermittent stimulation at an ISI of 3.2-4 s was replaced with continuous movements at $12 \mathrm{~Hz}(I S I=0.083 \mathrm{~s})$. More detailed characterization of the ISI effect would help design time-efficient stimulation protocols for both basic and clinical research by making it possible to determine the ISI yielding the maximum signalto-noise ratio (SNR) within a given recording time. Furthermore, knowledge of the ISI dependence of proprioceptive responses in healthy individuals could be utilized to study potential abnormalities in this dependence in various diseases (Andrade et al., 2016).

Here we recorded cortical responses to passive index-finger movements at various ISIs. Our aim was to accurately model the ISI dependence of the responses to learn about the temporal operating scales of the underlying cortical processes and to estimate the optimum ISI for assessing proprioceptive afference.

This article is protected by copyright. All rights reserved. 


\section{Materials and methods}

\section{Subjects}

We studied 15 healthy adults (9 men, 6 women; ages $22-38$ yrs, mean 29.9 yrs). All subjects were right-handed (score range 60-100, mean score 87) according to the Edinburgh handedness inventory (Oldfield, 1971). All had signed a written informed consent prior to participation. Participants received compensation for travel expenses and lost working hours. The study conformed to the Declaration of Helsinki and had a prior approval by the ethics committee of Aalto University.

\section{Experimental protocol}

Fig. 1 shows the MEG setup. The subjects were instructed to sit relaxed, resting their left hand on their thigh and their pronated right hand on the upper surface of a pneumatic artificial-muscle (PAM) stimulator (Piitulainen et al., 2015), placed on a table in front of them. The tip of the right index finger was taped (Leukoplast ${ }^{\circledR}$ medical tape) to the extremity of the vertically-oriented actuator of the PAM stimulator that generated transient flexion and extension movements primarily at the metacarpophalangeal joint of the finger. The subjects were not able to see the finger nor the PAM stimulator as their vision for that part of the visual field was blocked with a sheet of paper (Fig. 1).

Fig. 2A illustrates the timecourse of the movement stimuli at an ISI of $8 \mathrm{~s}$. Extension and flexion movements occurred alternately every $4 \mathrm{~s}$ (half of the ISI), meaning that one ISI included two movements and two volleys of proprioceptive afference. Fig. 2B shows the enlarged profiles of an extension and flexion movement. The movements were rapid in both directions, with about $100 \mathrm{~ms}$ from movement onset to the midpoint of the trajectory (Fig. 2B, dashed horizontal line).

The experiment comprised seven movement blocks, and one rest block. Movements were elicited at fixed ISIs of $0.5,1,2,4,8$, and $16 \mathrm{~s}$ for $\left.2 \mathrm{~min}\left(\mathrm{ISI}_{0.5}, \mathrm{ISI}_{1} \text { and ISI }\right)_{2}\right), 4 \mathrm{~min}\left(\mathrm{ISI}_{4}\right), 6 \mathrm{~min}\left(\mathrm{ISI}_{8}\right)$, or $2 \times 6$ min $\left(\mathrm{ISI}_{16}\right)$, corresponding to 45-240 movements depending on the ISI. The time between consecutive blocks was $\sim 2 \mathrm{~min}$. The order of the different movement blocks was randomized for each subject. In the rest block, which was always the last of the session, the subjects were sitting relaxed and eyes open for $5 \mathrm{~min}$, resting the right hand on the PAM stimulator as in the movement blocks. During the rest block no stimuli were applied and the data were used only to estimate the electromyogram (EMG) level during rest.

During the MEG recordings, the subjects fixated their gaze on a fixation cross displayed on a screen in front of them. They wore earplugs to avoid hearing any acoustic noise from the PAM stimulator.

\section{Recordings}

Magnetoencephalographic (MEG) signals were recorded with a 306-channel whole-scalp neuromagnetometer (Elekta Neuromag ${ }^{\mathrm{TM}}$, Elekta Oy, Helsinki, Finland) in a magnetically shielded room (Imedco AG, Hägendorf, Switzerland) at the MEG Core of Aalto Neurolmaging, Aalto University. Recording passband was $0.1-330 \mathrm{~Hz}$ and the sampling rate $1000 \mathrm{~Hz}$. The position of the subject's head inside the MEG helmet was continuously monitored by feeding current through five head-position coils attached to the scalp at frequencies well above the physiological frequencies of interest.

This article is protected by copyright. All rights reserved. 
Acceleration signals from passive finger movements were recorded with a 3-axis accelerometer (ADXL335 iMEMS Accelerometer, Analog Devices Inc., Norwood, MA, USA) attached to the nail of the right index finger. The filtered (passband $0.1-330 \mathrm{~Hz}$ ) acceleration signals were sampled at $1000 \mathrm{~Hz}$, time-locked to the MEG signals.

Surface EMG was monitored from right antebrachial muscles, with one active electrode on the flexor and another on the extensor side; the reference electrode was over the distal radial bone. EMG signals were filtered (passband $10-330 \mathrm{~Hz}$ ) and sampled at $1000 \mathrm{~Hz}$ time-locked to the MEG signals.

Anatomical magnetic resonance images (MRIs) were acquired with a General Electric Signa ${ }^{\circledR}$ 3T (Signa VH/i, General Electric, Milwaukee, WI, USA) or a MAGNETOM Skyra ${ }^{\circledR}$ 3T (Siemens Healthcare, Erlangen, Germany) whole-body MRI scanner at the AMI Centre of Aalto Neurolmaging, Aalto University.

\section{Data preprocessing}

A temporal extension of the signal space separation method (Taulu \& Simola, 2006) was applied off-line to the MEG signals (segment length $16 \mathrm{~s}$; correlation limit 0.9) to reduce artifacts and to correct for head movements. For each subject, signals representing different ISIs were transformed to the same head-coordinate system (mean over ISIs) to allow comparison between responses obtained at different ISIs.

Wide-band MEG signals were averaged with custom-made MATLAB ${ }^{\circledR}$ (MathWorks, Natick, MA, USA) scripts separately for each ISI and both movement directions, thus yielding 12 averaged responses for each subject. To find the proper triggering moment, we first filtered (passband 1-195 $\mathrm{Hz}$ ) the 3 orthogonal acceleration signals and combined them into a single signal by computing their Euclidean norm. Movement onset was determined, for all single movements, as the time point preceding the rise of acceleration above $10 \%$ of its maximum during that movement. To avoid any bias caused by rarely occurring artifacts in the acceleration signal, we finally defined the movement onset on the basis of the typical (most frequent) trigger-to-movement-onset latency, separately for each subject, ISI, and movement direction. We then averaged 39-244 (depending on the ISI) MEG epochs spanning from -500 to $1000 \mathrm{~ms}$ with respect to movement onset. All stimuli occurring during the first $4 \mathrm{~s}$ were discarded from the analysis to avoid inclusion of nonstationary responses. We also omitted epochs during which any of the MEG signals exceeded $3 \mathrm{pT}$ (magnetometers) or $0.7 \mathrm{pT} / \mathrm{cm}$ (gradiometers). Finally, the averaged MEG signals were low-pass filtered at $40 \mathrm{~Hz}$ and adjusted to a baseline from -150 to $0 \mathrm{~ms}$.

\section{Data analysis}

Source-space analysis

We modeled, separately for each subject's extension and flexion movements, the source of the most prominent deflection of the $\mathrm{ISI}_{8}$ response with an equivalent current dipole (ECD) using Elekta Neuromag ${ }^{\mathrm{TM}}$ software (Elekta Oy, Helsinki, Finland). The most prominent response peaked 70 $\mathrm{ms}$ after extension and $\sim 90 \mathrm{~ms}$ after flexion movements. The ECD was fitted to best explain the signals obtained from a fixed selection of 18 planar gradiometers (from nine sensor units) above the left sensorimotor cortex. The ECD obtained at $\mathrm{ISI}_{8}$ was then employed as a reference source also for

This article is protected by copyright. All rights reserved. 
the other ISIs. Subject 10 lacked a clear cortical response in the typical time window for $\mathrm{ISI}_{8}$ flexion, and in his case, the ECD for flexion movements was obtained from the $\mathrm{ISI}_{4}$ response.

The source waveform was plotted for each ISI, and its maximum value between 30 and $130 \mathrm{~ms}$ was extracted. These maximum source-strength values were individually normalized by dividing them by the individual's mean across ISIs. Then, the ISI dependence of the source strength, was modeled with the exponential saturation function

$$
A(I S I)=A_{\max }\left(1-e^{-\frac{I S I}{\tau}}\right)
$$

where $A$ is the normalized source strength, $A_{\max }$ is the highest $A$ that can be reached for arbitrarily long ISIs, and $\tau$ is the lifetime of the response. $A(I S I)$ was fitted to the group-mean data with the unconstrained nonlinear optimization function "fminsearch" implemented in MATLAB.

Within a fixed measurement time, prolongation of the ISI increases the response amplitude but simultaneously increases the noise level since a smaller number of stimuli $(n)$ can be collected in the given time. For stationary noise, the noise level decreases by a factor of $\sqrt{n} \sim \frac{1}{\sqrt{I S I}}$ with response averaging, and consequently, the SNR of the averaged response is proportional to $\left(1-e^{-\frac{I S I}{\tau}}\right) / \sqrt{I S I}$. This SNR value is at maximum when ISI $\approx 1.26 \tau$. In other words, $1.26 \tau$ is the optimum ISI within a fixed measurement time. To verify that noise was indeed stationary across ISIs, which would justify the use of this estimate, we evaluated the noise level separately for each subject and ISI. Noise was evaluated as the standard deviation of the differences at each time point between all single trials and the averaged response.

We performed a similar source-space analysis also with stimulus-number-matched data to control for the effect of the number of stimulus repetitions that also affects the SNR of the averaged response. The number of stimuli was higher at ISIs of $0.5,1$, and $2 \mathrm{~s}$ (with $\sim 60, \sim 120$, and $\sim 240$ responses averaged, respectively) compared with ISIs of 4, 8, and $16 s$ ( 45$)$. Here, when computing the averaged responses at $|S|_{0.5}-|S|_{2}$, we matched the number of single trials with that at $|S|_{8}$. Otherwise the analysis was identical to the description above.

\section{Sensor-space analysis}

To confirm that the results we obtain are not related to the method used to estimate source activity, we analyzed the responses to extension movements also in sensor-space. To do so, we first combined the signals from gradiometer pairs by calculating their vector sums. For each subject, we then selected the sensor (among the nine pairs used in the source analysis) showing the highest peak vector sum between 30 and $130 \mathrm{~ms}$ for most of the ISIs. We extracted the peak values in this sensor and time window (30-130 ms) and normalized them within subjects. The ISI dependence of these sensor-space-response amplitudes was then modeled with an exponential saturation function as described above for source strengths. We performed this analysis only for extension (and not flexion) movements due to the smaller variability of the responses across ISIs and subjects.

\section{EMG analysis}

EMG signals from the flexor and extensor muscles were bandpass-filtered from 20 to $295 \mathrm{~Hz}$, and root-mean-square ( $\mathrm{rms}$ ) values of whole-length EMG signals were computed for all movement and rest conditions. Similarly as in the averaging procedure, we discarded the data during the first

This article is protected by copyright. All rights reserved. 
stimuli and started the analysis from the onset of the first flexion movement included in the average. Additionally, we averaged rectified EMG signals from the same epochs as the MEG signals.

\section{Statistical analysis}

To compare the cortical sources for extension and flexion movements, we tested the effect of movement direction on (a) source location with a repeated-measures one-way multivariate analysis of variance (MANOVA; $x, y$, and $z$ coordinates as 3 individual dependent factors), (b) source orientation on a tangential plane with a two-tailed paired t-test, and (c) source strength at each ISI with two-tailed paired t-tests and Bonferroni correction for multiple comparisons. The effect of ISI on source strength was modeled with an exponential saturation function fitted to the data, as described in Source-space analysis section. Furthermore, we assessed the effects of movement direction and ISI on the peak latency of the source waveform with a repeatedmeasures two-way analysis of variance (ANOVA). Here we also tested the interaction between movement direction and ISI, but as no statistically significant interaction was found, we removed the interaction term from the final model. In the case of violations of the sphericity assumption in the ANOVAs, the degrees of freedom were corrected with the Greenhouse-Geisser procedure. For such ANOVAs we report uncorrected degrees of freedom together with the correction factor $\varepsilon$, as well as the corrected $F$ and $P$ values. The alpha level was set at 0.05 in all statistical tests. All statistical analyses were performed with IBM SPSS Statistics 22.

\section{Results}

We successfully recorded cortical responses to passive finger extension and flexion movements from all 15 subjects, at least at the 5 longest ISIs (1-16 s). At the shortest ISI of $0.5 \mathrm{~s}$, responses to extensions and flexions were not detected in 2 and 4 subjects, respectively.

\section{Movement characteristics}

Fig. 2B shows the displacement of the movement actuator for both extension and flexion movements at $\mathrm{ISI}_{8}$. During the first $\mathbf{5 0} \mathbf{~ m s}$ after movement onset-the part of the stimulus that is relevant for the studied cortical responses peaking at $\sim 70$ and $\sim 90 \mathrm{~ms}-$ the displacement was very similar across all ISIs: $1.2-1.3 \mathrm{~mm}$ for extensions and 0.7-0.9 $\mathrm{mm}$ for flexions, corresponding to mean velocities of 25-26 and 13-18 mm/s, respectively. The total range of movement was $3.9 \mathrm{~mm}$ at $|S|_{0.5}$ and increased with the ISI up to $5.5 \mathrm{~mm}$ at $|S|_{16}$. At $|S|_{0.5}$, the movements lasted through the entire extension and flexion phases ( $250 \mathrm{~ms}$ each), whereas at longer ISIs, the movements reached a plateau at $\mathbf{3 0 0} \mathrm{ms}$.

Figs. 2C-E show the averaged finger acceleration and surface EMG signals for one representative subject (S1) at ISI. The peak acceleration was obtained already at $\sim 5 \mathrm{~ms}$, and it was higher for extension than flexion movements (Fig. 2C); similar acceleration profiles were obtained across subjects. EMG activity was negligible $(\sim 1 \mu \mathrm{V})$ in both extensor and flexor muscles during the movements (Figs. 2D and E). In group analysis, the rms EMG level during the movement conditions did not exceed the rest level, indicating that subjects were able to remain relaxed during the stimulation as instructed. However, tiny stimulus-locked EMG responses were visible in 14/15 subjects after the flexions (Fig. 2D and E) but only in $1 / 15$ subjects after the extensions. These EMG

This article is protected by copyright. All rights reserved. 
responses peaked between 100 and 200 ms after the movement onset, and thus clearly later than the observed cortical responses.

\section{MEG responses, cortical sources, and effect of ISI}

Fig. 3 shows the spatial distribution (A) and ISI dependence (B) of the averaged responses, as well as the corresponding cortical sources (C) and source waveforms (D) of a representative subject (S1). Prominent deflections occurred in the left contralateral sensorimotor cortex, peaking at 78-85 ms for extensions and 92-96 ms for flexions (Fig. 3B). In this subject, as well as in the whole group (Fig. S1), the responses to extensions were earlier, stronger, narrower, and more consistent than those to flexions. For both movement directions, the response amplitudes increased as a function of ISI, from practically no visible responses at $\left.I S\right|_{0.5}$ to prominent responses at the longest ISIs.

The sources of the responses to both extensions and flexions (Fig. 3C) were located in the posterior bank of the central sulcus, in the "hand knob" area (Yousry et al., 1997). Also in the other subjects, sources were located in the SI cortex with the intracellular current during the main peak always pointing posteriorly. At group level, the source location and orientation did not differ between extension and flexion movements (main effect of movement direction on source location in repeated-measures one-way MANOVA: $F_{3,12}=0.18, P=0.91$; effect of movement direction on source orientation in two-tailed paired $t$-test: $t_{14}=1.8, P=0.097$ ). The group-median goodness-of-fit values and confidence volumes for the ECDs at $\left.I S\right|_{8}$ were $99.1 \%$ (range $96.4-99.7 \%$ ) and $0.2 \mathrm{~cm}^{3}(0.04-1.6$ $\mathrm{cm}^{3}$ ) for extensions and $96.5 \%(91.6-99.7 \%)$ and $1.4\left(0.1-7.3 \mathrm{~cm}^{3}\right)$ for flexions, respectively.

The ISI dependence was similar for source strengths (Fig. 3D) as for the original averaged responses (Fig. 3B). The source strengths peaked, at group level, on average $19 \mathrm{~ms}$ earlier for extensions (mean \pm SD $75 \pm 17 \mathrm{~ms}$; range $47-125 \mathrm{~ms}$ ) than flexions ( $94 \pm 23 \mathrm{~ms} ; 32-130 \mathrm{~ms}$ ), whereas the peak latencies did not differ across ISIs (main effects of movement direction and ISI, respectively, on peak latency in repeated-measures two-way ANOVA: $F_{1,14}=26, P=0.00017$ and $F_{5,70}$ $=2.0, \varepsilon=0.58, P=0.13)$. Furthermore, the sources were $46-163 \%$ stronger for extension than flexion movements at $\left.I S\right|_{1}-|S|_{16}$, whereas at $I S I_{0.5}$ no difference was observed between movement directions $\left(P_{\text {corrected }}<0.05\right.$ for $\mid \mathrm{ISI}_{1}-\mathrm{ISI}_{16}$ and $P_{\text {uncorrected }}=0.51$ for $\mathrm{ISI}_{0.5}$; two-tailed paired $t$-tests with Bonferroni correction).

Fig. 4 shows the group-level ISI effect on source strengths for both extensions and flexions. The source strengths increased approximately 3 -fold for extensions and 6 -fold for flexions when the ISI was prolonged from 0.5 to $8 \mathrm{~s}$, and then the responses plateaued. This relationship was closely modeled with an exponential saturation function (see Source-space analysis section of Materials and methods), with response lifetimes $(\tau)$ and maximum source strengths ( $A_{\max } ;$ normalized within subjects) of $1.3 \mathrm{~s}$ and 1.3 for extension and $2.2 \mathrm{~s}$ and 1.5 for flexion movements. As the noise levels in the single-trial MEG signals were very similar across ISIs (group averages between 62 and 64 $\mathrm{fT} / \mathrm{cm}$ at all ISIs), the optimum ISI, maximizing SNR in a given measurement time, could be estimated simply as $1.26 \tau$ yielding $1.7 \mathrm{~s}$ for extension and $2.8 \mathrm{~s}$ for flexion movements. Based on these results, the optimum ISI $(1.26 \tau)$ that maximizes SNR in a given measurement time is $1.7 \mathrm{~s}$ for extension and $2.8 \mathrm{~s}$ for flexion movements. The optimum ISIs were similar (1.5 s for extensions and $2.0 \mathrm{~s}$ for flexions) also when estimated from the stimulus-number-matched data. For sensor-spaceresponse amplitudes (shown for extension movements in Fig. 4, left, dashed curve), the ISI dependence was closely similar to that for the source-space data (solid curve).

This article is protected by copyright. All rights reserved. 


\section{Discussion}

In the current study, passive finger movements elicited prominent responses in the contralateral sensorimotor cortex in accordance with earlier findings (Xiang et al., 1997; Lange et al., 2001; Alary et al., 2002; Druschky et al., 2003; Woldag et al., 2003; Onishi et al., 2013; Piitulainen et al., 2013, 2015). The corresponding cortical sources peaked $\sim 70 \mathrm{~ms}$ after extension movements and $\sim 90$ ms after flexion movements. Source strengths were markedly increased when the ISI was prolonged from 0.5 to $8 \mathrm{~s}$. Increasing the ISI further to $16 \mathrm{~s}$ did not any more increase the source strengths.

\section{Origin of passive-movement-evoked responses}

According to prior evidence, EEG/MEG responses to passive movements are primarily triggered by proprioceptive afference, whereas the role of tactile afference from cutaneous receptors is minor (Starr et al., 1981; Abbruzzese et al., 1985; Druschky et al., 2003; Piitulainen et al., 2013; Bourguignon et al., 2015). For example, EEG responses to passive plantar flexions of the ankle are not affected by cutaneous anesthesia of the foot (induced with a cuff above the ankle) but are attenuated by blocking the flow of proprioceptive afference in the peroneal nerve either with pressure or a local anesthetic (Starr et al., 1981). Similarly, cutaneous anesthesia has no effect on EEG responses to passive wrist extensions (Abbruzzese et al., 1985) or finger flexions (Mima et al., 1996). Furthermore, MEG responses to passive finger movements differ from tactile responses in their longer peak latencies and higher amplitudes (Druschky et al., 2003). Finally, decisive evidence for the dominance of proprioceptive afference in movement-evoked cortical responses has come from recent MEG studies (Piitulainen et al., 2013; Bourguignon et al., 2015) exploring the coupling between peripheral movements and cortical activity by means of corticokinematic coherence (CKC; Bourguignon et al., 2011). CKC represents the steady-state counterpart of the movement-evoked cortical responses. It peaks in the primary sensorimotor cortex contralateral to the movements and is considerably (2.7-15.5 times) stronger in the afferent than in the efferent direction (Bourguignon et al., 2015). Concomitant tactile stimulation (by letting the moving index finger touch the table), increased the afferent coherence by up to $40 \%$ (Bourguignon et al., 2015) but even in this case, the proprioceptive signaling was the leading contributor to the coherent cortical activity. The dominant role of proprioceptive afferents to the elicited cortical signals is also strongly supported by the similarity of CKC for both active and passive finger movements (Piitulainen et al., 2013).

Muscle receptors are the most important proprioceptors for kinesthesia, the sense of movement (for a review, see Proske \& Gandevia, 2012). However, also cutaneous stretch receptors - that are located around joints, especially in fingers, but distinct from the cutaneous pressure receptors that mediate tactile sensations (Gardner \& Johnson, 2013)-contribute to kinesthetic percepts (Collins et al., 2005). The present data do not allow to determine the relative contributions of these different proprioceptor types on the observed cortical responses.

In monkeys, muscle receptors project to areas $3 \mathrm{a}$ and 2 of the SI cortex (Burchfiel \& Duffy, 1972; Schwarz et al., 1973), whereas areas 3b and 1 receive mostly tactile input (for a review, see Kaas, 1993). Some MI neurons are activated by passive movements as well, both in monkeys (Lucier et al., 1975) and humans (Goldring \& Ratcheson, 1972). In the current study, the responses to passive finger movements were adequately modeled with posteriorly-pointing dipoles in the contralateral primary sensorimotor cortex, in good agreement with previous MEG studies (Xiang et al., 1997; Lange et al., 2001; Alary et al., 2002; Druschky et al., 2003). More specifically, our results

This article is protected by copyright. All rights reserved. 
indicated that the sources were located in the posterior bank of the central sulcus, corresponding to areas $3 \mathrm{a} / 3 \mathrm{~b}$ of the $\mathrm{SI}$ cortex. The current study did not allow differentiation between these areas, although earlier knowledge about the functional organization of the SI cortex would suggest area 3a rather than 3b as the origin of proprioceptive responses (Kaas, 1993). In the posterior bank of the central sulcus, a posterior source orientation implies intracellular currents flowing from the cortical surface towards deeper layers. Such currents are best explained by excitation in the superficial cortical layers.

\section{ISI dependence of cortical reactivity}

The observed ISI dependence of the passive-movement-elicited cortical responses is in line with previous EEG studies using transient passive movements (Starr et al., 1981; Abbruzzese et al., 1985) and with a recent MEG study using both transient and continuous passive movements (Piitulainen et al., 2015). To our knowledge, however, ours is the first study to characterize this ISI dependence in detail and quantify the lifetime of these cortical responses. Our exponential saturation function that modeled well the ISI dependence of the response strength is similar to the models proposed previously for auditory (Lu et al., 1992a), visual (Uusitalo et al., 1996), and nociceptive (Raij et al., 2003) cortical responses. Based on our model, we were also able to estimate the optimum ISI for proprioceptive stimulation to reach maximum SNR in a given measurement time. This optimization was a compromise between response amplitude, which increases, and the number of stimuli, which decreases as a function of ISI. A similar ISIoptimization approach has been previously applied to visual (Ahlfors et al., 1993) and nociceptive (Raij et al., 2003) MEG responses.

Cortical activation by external sensory stimulation is typically followed by a recovery period of lowered reactivity and decreased response amplitudes to subsequent stimuli. These changes cannot be explained by neuronal fatigue, but might instead be the result of active inhibition of a subset of the neuronal populations contributing to the response (Loveless et al., 1989). The duration of the recovery period is characterized by the response lifetime $\tau$, and it varies depending on the cortical area as well as the specific neuronal population within that area (for a review, see Hari et al., 2010). Responses to visual stimuli in early occipital areas recover faster than those in higher-order temporal, parietal and frontal areas (Uusitalo et al., 1996), and a similar division is observed between the auditory cortices (Lu et al., 1992b; Sams et al., 1993).

Late EEG responses at $\sim 460$ ms after painful stimulation of the nasal mucosa are attenuated at ISIs as long as $60 \mathrm{~s}$, as are also subjects' ratings of stimulus intensity (Hummel \& Kobal, 1999). However, this attenuation largely depends on peripheral adaptation mechanisms (Hummel et al., 1996).

The current results indicate that at ISIs up to $4 \mathrm{~s}$, and even longer, the SI cortex does not fully recover between consecutive volleys of proprioceptive afference, whereas ISIs of $8 \mathrm{~s}$ and $16 \mathrm{~s}$ yield practically the maximum responses. Closer analysis revealed response lifetimes of $1.3 \mathrm{~s}$ for extension and $2.2 \mathrm{~s}$ for flexion movements. Thus, the recovery rate of proprioceptive SI responses was comparable to those reported previously for the supratemporal auditory N100m response (Hari et al., 1982; Lu et al., 1992a) and the $100-m s$ SIl response to electrical median nerve stimulation (Hari et al., 1993). However, due to the biphasic stimulation-containing one extension and one flexion movement per cycle-the interval between consecutive movements was in our study only half of the applied ISI. Thus our quantification of the response lifetimes is based on the assumption of independence of proprioceptive afference from extensor and flexor muscles. If, however, these

This article is protected by copyright. All rights reserved. 
afferent volleys would interact in the cortex, or along the afferent pathways, then proprioceptive SI responses would in fact recover by a factor of 2 faster than estimated by the current study. Further investigations of such interactions are required to elucidate this question.

The observed response lifetimes indicate that an ISI of 1.5-3 s for passive-finger-movement stimulation maximizes the SNR of the averaged cortical responses in a given measurement time. This information may be utilized in future clinical and research protocols to save recording time and ensure repeatability between measurements. The optimization is an important step when aiming at a standardized clinical tool to diagnose impairments of proprioceptive function, which may occur for example due to stroke, cerebellar degeneration, myelopathies, cerebral palsy, and neuropsychiatric conditions. Our results can also be valuable for future developmental studies (Uppal et al., 2016) as well as for exploring the link between electrophysiological markers and genetic susceptibility to diseases that affect the sensorimotor system.

\section{Differences in cortical activations for extension and flexion movements}

Although both extension and flexion movements strongly activated the contralateral SI cortex, the evoked responses differed; the extensions evoked earlier, stronger, and more consistent cortical responses with more clearly dipolar field patterns. One possible explanation for these differences is that the extensions were slightly faster than the flexions, due to mechanical properties of the PAM stimulator. Furthermore, because of the initial extended position of the finger, the extensions caused stronger stretch in the flexor muscles than the flexions in the extensors, better activating the proprioceptors (for a review, see Proske \& Gandevia, 2012).

\section{Conclusions}

Proprioceptive stimulation using passive index-finger movements strongly activated the contralateral primary somatosensory cortex with the most prominent response peaking at $\sim 70 \mathrm{~ms}$ for extension and $\sim 90 \mathrm{~ms}$ for flexion movements. The strength of the cortical sources was markedly enhanced when the ISI was prolonged as observed previously for other sensory modalities. Detailed characterization of this ISI effect indicated an optimum ISI of 1.5-3 s for proprioceptive stimulation to maximize the SNR in a fixed measurement time. Our results can be vastly utilized in future studies exploring proprioceptive processing in different subject groups, as well as in designing standardized stimulation protocols for both basic and translational research.

\section{Acknowledgements}

This work was supported by the Academy of Finland (Grants \#131483 and \#263800 to Riitta Hari and Grants \#266133 and \#296240 to Harri Piitulainen), Tekes - the Finnish Funding Agency for Technology and Innovation (Grant 1104/10), the European Research Council (Advanced Grant \#232946 to Riitta Hari), the Emil Aaltonen Foundation (Eero Smeds), and the Research Programs Unit of the University of Helsinki (Eero Smeds). The funding sources were not involved in the study design; in the collection, analysis and interpretation of data; in the writing of the report; nor in the decision to submit the article for publication. We thank Helge Kainulainen for technical support.

This article is protected by copyright. All rights reserved. 


\section{Conflict of interest}

None of the authors have potential conflicts of interest to be disclosed.

\section{Author contributions}

$E S, H P, M B$, and $R H$ designed the study. ES, HP, and MB collected the data. All authors participated in analyzing and interpreting the data and in writing the manuscript.

\section{Abbreviations}

$A$, strength (amplitude) of cortical source normalized within subjects; $A_{\max }$, maximum $A$ that can be reached for arbitralily long ISIs; ANOVA, analysis of variance; CKC, corticokinematic coherence; ECD, equivalent current dipole; EEG, electroencephalography; EMG, electromyography; $I S I$, interstimulus interval (subscript number indicates length of ISI in seconds, e.g. ISI 8 ); MANOVA, multivariate analysis of variance; MEG, magnetoencephalography; $\mathrm{MI}$, primary motor; $\mathrm{MRI}$, magnetic resonance image/imaging; $n$, number of stimulus repetitions in an experiment; PAM, pneumatic artificial muscle; rms, root mean square; SEF, somatosensory evoked field; SI, primary somatosensory; SII, secondary somatosensory; SNR, signal-to-noise ratio; $\tau$, response lifetime.

\section{Data accessibility}

The ethical approval for our study by the ethics committee of Aalto University does not allow public sharing of the original data.

\section{References}

Abbruzzese, G., Berardelli, A., Rothwell, J.C., Day, B.L. \& Marsden, C.D. (1985) Cerebral potentials and electromyographic responses evoked by stretch of wrist muscles in man. Exp Brain Res, 58, 544-551.

Ahlfors, S.P., Ilmoniemi, R.J. \& Portin, K. (1993) The effect of stimulation rate on the signal-to-noise ratio of evoked responses. Electroencephalogr Clin Neurophysiol, 88, 339-342.

Alary, F., Simões, C., Jousmäki, V., Forss, N. \& Hari, R. (2002) Cortical activation associated with passive movements of the human index finger: an MEG study. Neuroimage, 15, 691-696.

Andrade, G.N., Butler, J.S., Peters, G.A., Molholm, S. \& Foxe, J.J. (2016) Atypical visual and somatosensory adaptation in schizophrenia-spectrum disorders. Transl Psychiatry, 6, e804.

Bourguignon, M., De Tiège, X., Op de Beeck, M., Pirotte, B., Van Bogaert, P., Goldman, S., Hari, R. \& Jousmäki, V. (2011) Functional motor-cortex mapping using corticokinematic coherence. Neuroimage, 55, 1475-1479.

This article is protected by copyright. All rights reserved. 
Bourguignon, M., Piitulainen, H., De Tiège, X., Jousmäki, V. \& Hari, R. (2015) Corticokinematic coherence mainly reflects movement-induced proprioceptive feedback. Neuroimage, 106, 382-390.

Burchfiel, J.L. \& Duffy, F.H. (1972) Muscle afferent input to single cells in primate somatosensory cortex. Brain Res, 45, 241-246.

Collins, D.F., Refshauge, K.M., Todd, G. \& Gandevia, S.C. (2005) Cutaneous Receptors Contribute to Kinesthesia at the Index Finger, Elbow, and Knee. J Neurophysiol, 94, 1699-1706.

Druschky, K., Kaltenhauser, M., Hummel, C., Druschky, A., Huk, W.J., Neundorfer, B. \& Stefan, H. (2003) Somatosensory evoked magnetic fields following passive movement compared with tactile stimulation of the index finger. Exp Brain Res, 148, 186-195.

Gardner, E. \& Johnson, K. (2013) The Somatosensory System: Receptors and Central Pathways. In Kandel, E.R., Schwartz, J.H., Jessell, T.M., Siegelbaum, S.A. \& Hudspeth, A.J. (eds), Principles of neural science. McGraw-Hill, New York, pp. 475-497.

Goldring, S. \& Ratcheson, R. (1972) Human motor cortex: sensory input data from single neuron recordings. Science, 175, 1493-1495.

Hari, R., Kaila, K., Katila, T., Tuomisto, T. \& Varpula, T. (1982) Interstimulus interval dependence of the auditory vertex response and its magnetic counterpart: implications for their neural generation. Electroencephalogr Clin Neurophysiol, 54, 561-569.

Hari, R., Karhu, J., Hämäläinen, M., Knuutila, J., Salonen, O., Sams, M. \& Vilkman, V. (1993) Functional organization of the human first and second somatosensory cortices: a neuromagnetic study. Eur J Neurosci, 5, 724-734.

Hari, R., Parkkonen, L. \& Nangini, C. (2010) The brain in time: insights from neuromagnetic recordings. Ann N Y Acad Sci, 1191, 89-109.

Hummel, T. \& Kobal, G. (1999) Chemosensory event-related potentials to trigeminal stimuli change in relation to the interval between repetitive stimulation of the nasal mucosa. Eur Arch Otorhinolaryngol, 256, 16-21.

Hummel, T., Schiessl, C., Wendler, J. \& Kobal, G. (1996) Peripheral electrophysiological responses decrease in response to repetitive painful stimulation of the human nasal mucosa. Neurosci Lett, 212, 37-40.

Kaas, J.H. (1993) The functional organization of somatosensory cortex in primates. Ann Anat, 175, 509-518.

Lange, R., Nowak, H., Haueisen, J. \& Weiller, C. (2001) Passive finger movement evoked fields in magnetoencephalography. Exp Brain Res, 136, 194-199.

Loveless, N., Hari, R., Hämäläinen, M. \& Tiihonen, J. (1989) Evoked responses of human auditory cortex may be enhanced by preceding stimuli. Electroencephalogr Clin Neurophysiol, 74, 217227.

This article is protected by copyright. All rights reserved. 
Lu, Z.L., Williamson, S.J. \& Kaufman, L. (1992a) Behavioral lifetime of human auditory sensory memory predicted by physiological measures. Science, 258, 1668-1670.

Lu, Z.L., Williamson, S.J. \& Kaufman, L. (1992b) Human auditory primary and association cortex have differing lifetimes for activation traces. Brain Res, 572, 236-241.

Lucier, G.E., Ruegg, D.C. \& Wiesendanger, M. (1975) Responses of neurones in motor cortex and in area $3 \mathrm{~A}$ to controlled stretches of forelimb muscles in cebus monkeys. J Physiol, 251, 833-853.

Mima, T., Terada, K., Maekawa, M., Nagamine, T., Ikeda, A. \& Shibasaki, H. (1996) Somatosensory evoked potentials following proprioceptive stimulation of finger in man. Exp Brain Res, 111, 233-245.

Mäkelä, J.P., Ahonen, A., Hämäläinen, M., Hari, R., Ilmoniemi, R., Kajola, M., Knuutila, J., Lounasmaa, O.V., McEvoy, L., Salmelin, R., Salonen, O., Sams, M., Simola, J., Tesche, C. \& Vasama, J.-P. (1993) Functional differences between auditory cortices of the two hemispheres revealed by whole-head neuromagnetic recordings. Hum Brain Mapp, 1, 48-56.

Oldfield, R.C. (1971) The assessment and analysis of handedness: the Edinburgh inventory. Neuropsychologia, 9, 97-113.

Onishi, H., Sugawara, K., Yamashiro, K., Sato, D., Suzuki, M., Kirimoto, H., Tamaki, H., Murakami, H. \& Kameyama, S. (2013) Neuromagnetic activation following active and passive finger movements. Brain Behav, 3, 178-192.

Papakostopoulos, D., Cooper, R. \& Crow, H.J. (1974) Cortical potentials evoked by finger displacement in man. Nature, 252, 582-584.

Piitulainen, H., Bourguignon, M., De Tiège, X., Hari, R. \& Jousmäki, V. (2013) Corticokinematic coherence during active and passive finger movements. Neuroscience, 238, 361-370.

Piitulainen, H., Bourguignon, M., Hari, R. \& Jousmäki, V. (2015) MEG-compatible pneumatic stimulator to elicit passive finger and toe movements. Neuroimage, 112, 310-317.

Proske, U. \& Gandevia, S.C. (2012) The proprioceptive senses: their roles in signaling body shape, body position and movement, and muscle force. Physiol Rev, 92, 1651-1697.

Raij, T.T., Vartiainen, N.V., Jousmäki, V. \& Hari, R. (2003) Effects of interstimulus interval on cortical responses to painful laser stimulation. J Clin Neurophysiol, 20, 73-79.

Rodin, E., Wasson, S. \& Porzak, J. (1969) Objective evaluation of joint sense and touch in the human. Neurology, 19, 247-257.

Sams, M., Hari, R., Rif, J. \& Knuutila, J. (1993) The Human Auditory Sensory Memory Trace Persists about 10 sec: Neuromagnetic Evidence. J Cogn Neurosci, 5, 363-370.

Schwarz, D.W., Deecke, L. \& Fredrickson, J.M. (1973) Cortical projection of group I muscle afferents to areas 2, 3a, and the vestibular field in the rhesus monkey. Exp Brain Res, 17, 516-526.

This article is protected by copyright. All rights reserved. 
Scott, S.H. (2012) The computational and neural basis of voluntary motor control and planning. Trends Cogn Sci, 16, 541-549.

Shibasaki, H., Barrett, G., Halliday, E. \& Halliday, A.M. (1980) Cortical potentials following voluntary and passive finger movements. Electroencephalogr Clin Neurophysiol, 50, 201-213.

Starr, A., McKeon, B., Skuse, N. \& Burke, D. (1981) Cerebral potentials evoked by muscle stretch in man. Brain, 104, 149-166.

Taulu, S. \& Simola, J. (2006) Spatiotemporal signal space separation method for rejecting nearby interference in MEG measurements. Phys Med Biol, 51, 1759-1768.

Tiihonen, J., Hari, R. \& Hämäläinen, M. (1989) Early deflections of cerebral magnetic responses to median nerve stimulation. Electroencephalogr Clin Neurophysiol, 74, 290-296.

Uppal, N., Foxe, J.J., Butler, J.S., Acluche, F. \& Molholm, S. (2016) The neural dynamics of somatosensory processing and adaptation across childhood: a high-density electrical mapping study. J Neurophysiol, 115, 1605-1619.

Uusitalo, M.A., Williamson, S.J. \& Seppä, M.T. (1996) Dynamical organisation of the human visual system revealed by lifetimes of activation traces. Neurosci Lett, 213, 149-152.

Wikström, H., Huttunen, J., Korvenoja, A., Virtanen, J., Salonen, O., Aronen, H. \& Ilmoniemi, R.J. (1996) Effects of interstimulus interval on somatosensory evoked magnetic fields (SEFs): a hypothesis concerning SEF generation at the primary sensorimotor cortex. Electroencephalogr Clin Neurophysiol, 100, 479-487.

Woldag, H., Waldmann, G., Schubert, M., Oertel, U., Maess, B., Friederici, A. \& Hummelsheim, H. (2003) Cortical neuromagnetic fields evoked by voluntary and passive hand movements in healthy adults. J Clin Neurophysiol, 20, 94-101.

Xiang, J., Hoshiyama, M., Koyama, S., Kaneoke, Y., Suzuki, H., Watanabe, S., Naka, D. \& Kakigi, R. (1997) Somatosensory evoked magnetic fields following passive finger movement. Brain Res Cogn Brain Res, 6, 73-82.

Yousry, T.A., Schmid, U.D., Alkadhi, H., Schmidt, D., Peraud, A., Buettner, A. \& Winkler, P. (1997) Localization of the motor hand area to a knob on the precentral gyrus. A new landmark. Brain, 120, 141-157.

\section{Figure captions}

Figure 1. Measurement setting. (A) Subject sitting with his head inside the MEG helmet and his right index finger attached to the actuator of the PAM stimulator. (B) Close-up of a subject's hand resting on the stimulator, with the index finger in the extended position.

This article is protected by copyright. All rights reserved. 
Figure 2. Passive movements and EMG signals. (A) Displacement of the movement actuator during 1 min of stimulation at the 8-s ISI. (B) One movement cycle in focus. (C) Averaged finger acceleration (Euclidean norm of 3 signals) for extension ( $N=45)$ and flexion ( $N=46$ ) movements for Subject 1 (ISI $8 \mathrm{~s})$. (D, E) Averaged rectified EMG signals from antebrachial extensor and flexor muscles of Subject 1 (ISI 8 s). A small stimulus-locked EMG response peaks in both muscles $130 \mathrm{~ms}$ after the flexion movement (right traces), whereas no response is visible after the extension movement (left traces).

Figure 3. MEG responses and cortical sources evoked by passive extension and flexion movements of the right index finger in Subject 1. (A) Distribution of planar-gradiometer responses to extension movements of the right index finger at the 8-S ISI. (B) Responses, from the channel marked in (A) for both extension and flexion movements at all ISIs. (C) ECDs, fitted to the peak responses to extension (white) and flexion (black) movements at the 8-s ISI, superimposed to the subject's MRI. (D) Source waveforms following extension and flexion movements at all ISIs. In (B) and (D), solid vertical lines indicate movement onset, and dashed vertical lines at $|S|_{0.5}$ indicate the onset of the opposite movement occurring at $250 \mathrm{~ms}$.

Figure 4. ISI dependence of proprioceptive cortical responses. Normalized source strength (dots and error bars; mean \pm SEM over subjects) plotted as a function of ISI, separately for extension and flexion movements. The solid curves illustrate the function $A(I S I)=A_{\max }\left(1-e^{-\frac{I S I}{\tau}}\right)$, fitted to the group-mean values, where $A_{\max }$ is the highest $A$ reached for arbitrarily long ISIs, and $\tau$ is the lifetime of the response. The estimated lifetimes $\tau$ are $1.3 \mathrm{~s}$ (extension) and $2.2 \mathrm{~s}$ (flexion). Note that the $\mathrm{y}$ axes are scaled to $A_{\max }$. The vertical lines indicate the optimum ISIs maximizing the SNR in a fixed measurement time. The dashed curve in the left panel, plotted on top of the solid curve, illustrates the shape of the ISI effect estimated from sensor-space responses to extension movements.

This article is protected by copyright. All rights reserved. 

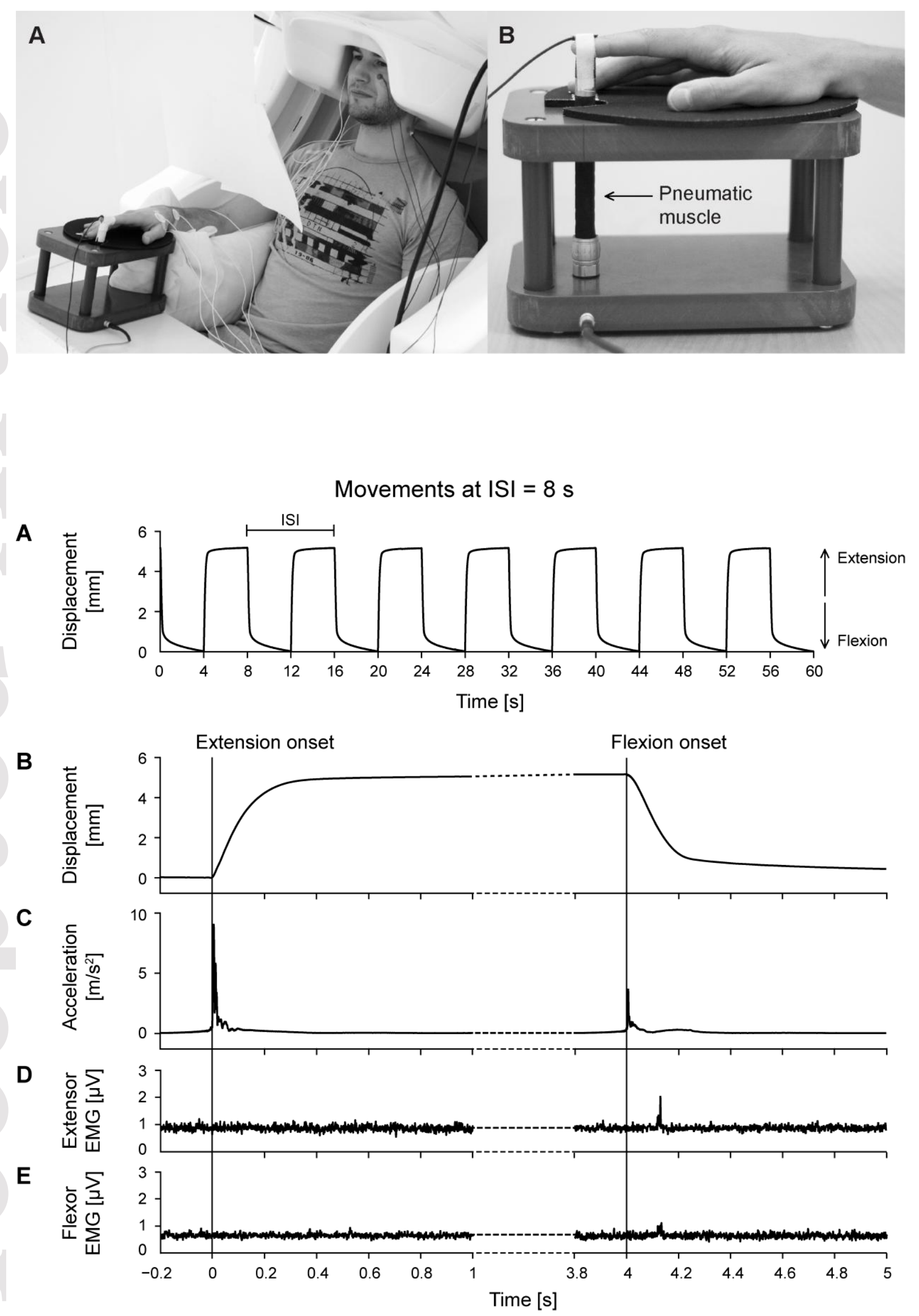

This article is protected by copyright. All rights reserved. 

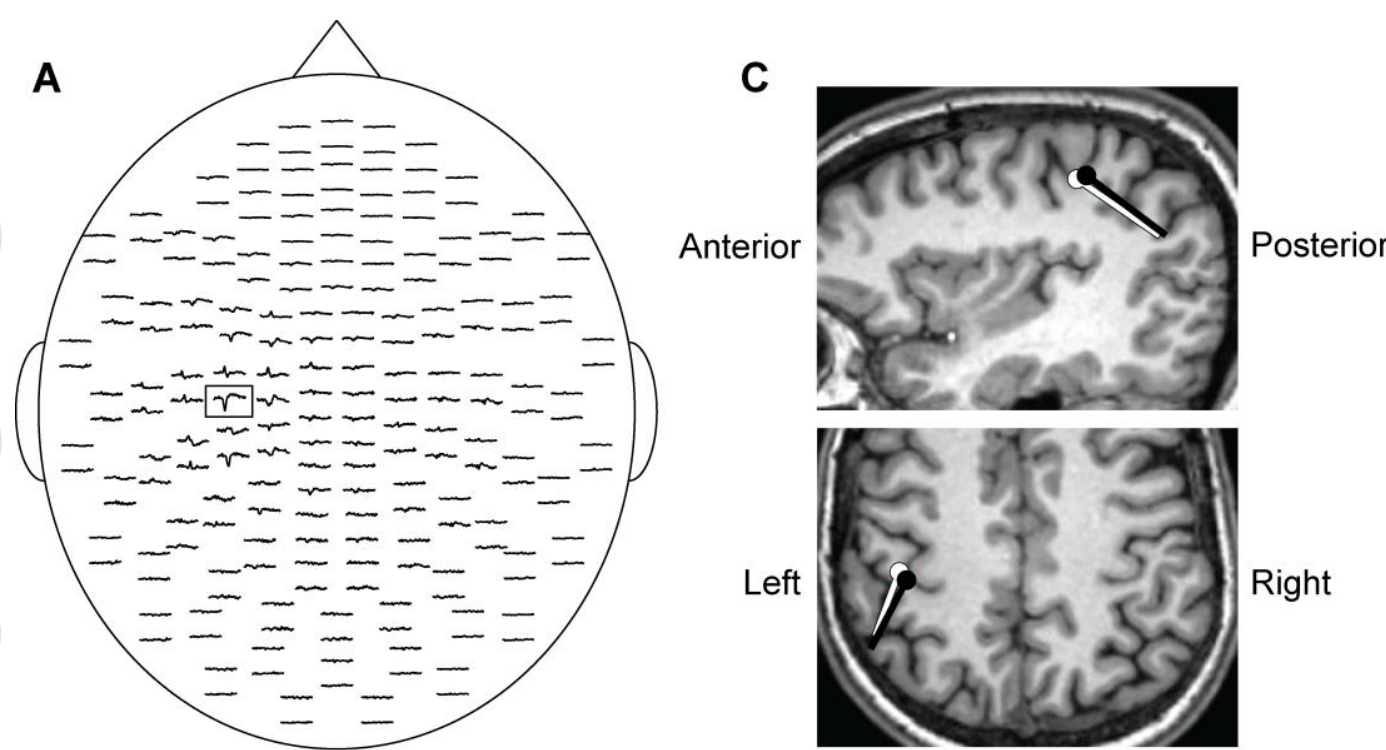

B Single-channel responses

D Source waveforms

ISI [s] Extension Flexion
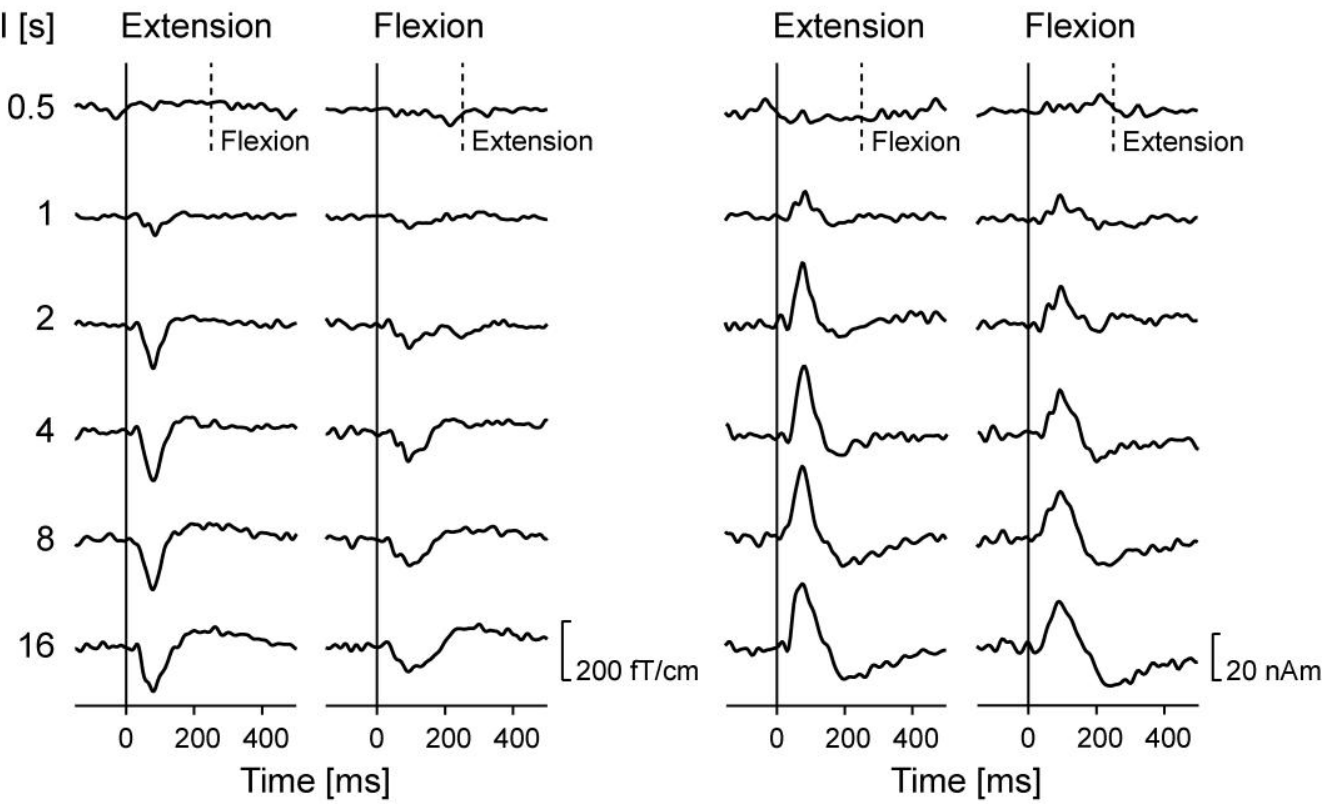

This article is protected by copyright. All rights reserved. 
Extension

Flexion
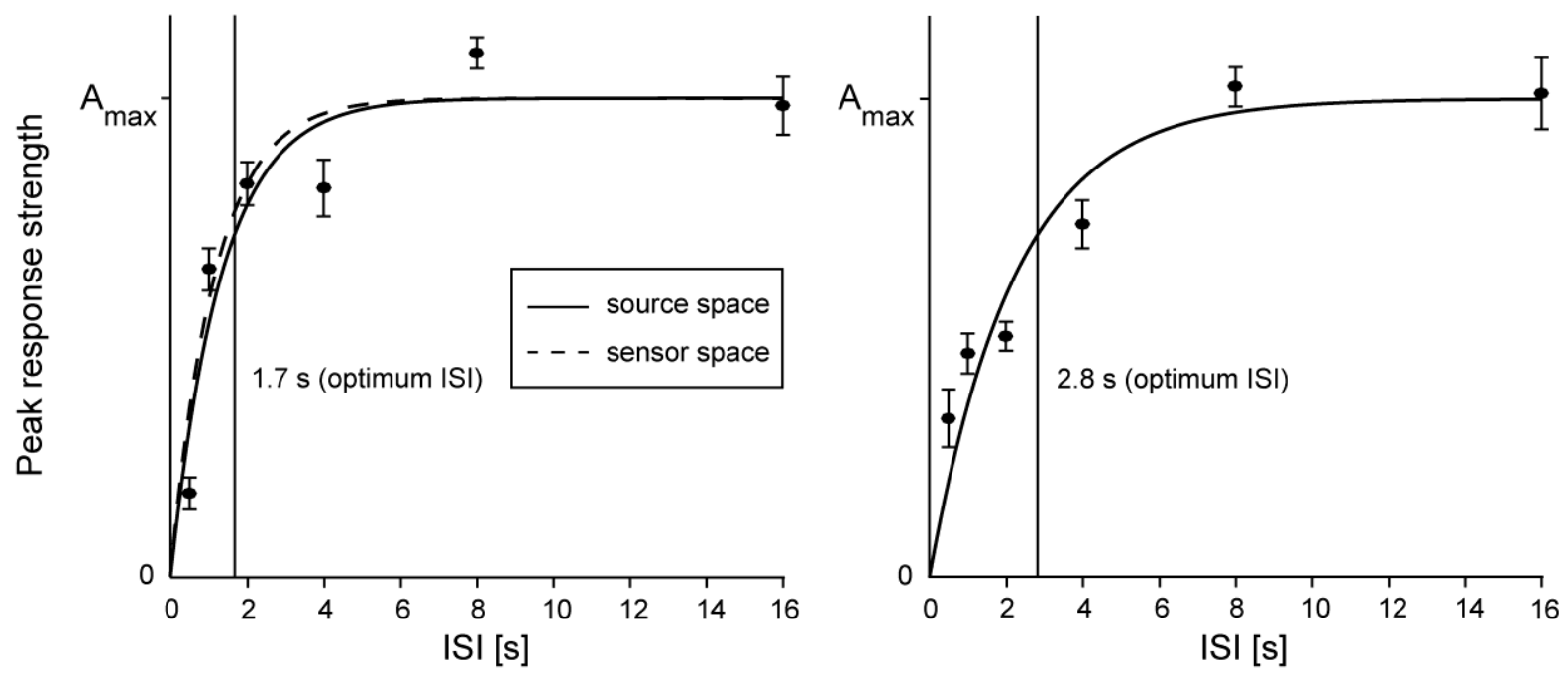

This article is protected by copyright. All rights reserved. 